Casos Clínicos

Arch. Esp. Urol. 2010; 63 (7): 547-549

\section{NEOPLASIA EPITELIAL RENAL ASOCIADA A LA ENFERMEDAD DE KIMURA. PRESENTACIÓN DE UN CASO}

\author{
Hilário Antonio de Castro Júnior, Marco Túlio Coelho \\ Lasmar, Eugenio Augusto Costa de Souza, Juliano \\ Alves Figueiredo, Breno Diniz Nogueira y Fernando \\ Carboni Tardelli.
}

Hospital Felício Rocho. Belo Horizonte. Minas Gerais. Brasil.

Resumen.- OBJETIVO: Presentar un caso de enfermedad de Kimura asociada a la neoplasia de riñón.

La enfermedad de Kimura se trata de una enfermedad inflamatoria rara, que se presenta comúnmente con linfonodos aumentados y masas en regiones de la cabeza y del cueIlo. El diagnóstico diferencial debe incluir linfomas, linfadenopatía reaccional a medicinas o parásitos. En masas y linfonodos aumentados se puede encontrar algún nódulo sugerente de neoplasia. Existe un caso en la literatura que describe a la Enfermedad de Kimura asociada a la neoplasia de riñón.

\section{CORRESPONDENCIA}

Hilário Antônio de Castro Júnior Hospital Felício Rocho

Belo Horizonte. Minas Gerais. (Brasil).

hilario.castro@sbu.org.br

Aceptado para publicar: 15 de octubre 2009.
MÉTODO: Se presenta el caso de una paciente joven, con nodulaciones intra y retroperitoneales, incluyendo una de ellas en el polo inferior del riñón izquierdo. Tras el período de tratamiento con corticoides, la tomografía computadorizada del abdomen confirmó la presencia de la lesión, con características de malignidad. Se realizó nefrectomía parcial izquierda y la evolución postoperatoria ha sido favorable.

RESULTADO: La paciente se encuentra sin manifestaciones de la Enfermedad de Kimura y sin signos de recidiva de la neoplasia 18 meses después de la operación.

CONCLUSIÓN: La enfermedad de Kimura está asociada a algunas neoplasias que deben ser investigadas por el equipo médico. La nefrectomía parcial parece ser una buena opción terapéutica para nódulos neoplásicos bien definidos y ubicados en polos renales.

Palabras clave: Enfermedad de Kimura. Eosinofília. Linfadenopatía eosinofilica. Neoplasia renal. Cáncer renal. Linfonodomegalia.

Summary.- OBJECTIVE: To present a case of Kimura disease associated with kidney epithelial neoplasia.

Kimura disease is a rare inflammatory disease, which usually presents with enlarged lymph nodes and masses in the head and neck regions. The differential diagnosis should include lymphomas, reaction lymphadenopathy to drugs or parasites. Amid the masses and enlarged lymph nodes a nodule suggestive of neoplasm can be found. There is a case, in the literature that describes Kimura Disease associated with kidney neoplasm.

METHOD: We present the case of a young female patient who showed intra and retroperitoneal nodulations, including one in the lower pole of left kidney. After the period of treatment with corticoids, computed tomography of abdomen confirmed the permanence of the lesion with features of malignancy. A partial left nephrectomy was performed, and postoperative evolution was favorable.

RESULT: The patient is without signs of Kimura Disease and no signs of recurrence of the fumor after a period of 18 months from surgery.

CONCLUSION: Kimura disease is associated with some neoplasias that should be investigated by the medical team. Partial nephrectomy seems a good therapeutic option for well-defined neoplastic nodules located on renal poles.

Keywords: Kimura Disease. Eosinophilia. Eosinophilic lynphadenopathy. Kidney Neoplasms. Kidney Cancer. Lymphadenitis. 


\section{INTRODUCCIÓN}

La Enfermedad de Kimura es una rara condición inflamatoria que cursa con lesiones nodulares subcutáneas y linfadenomegalia predominante de cabeza y cuello, como aumento de las glándulas salivares (1). Predomina en pacientes del sexo masculino y asiáticos, en la segunda y tercera décadas de vida. La enfermedad se caracteriza histológicamente por la proliferación fibrocolágena, vascular e inflamatoria, con abundante infiltrado eosinofílico (2). El tratamiento de la enfermedad se hace esencialmente con el uso de corticoides. La Enfermedad de Kimura asociada a la neoplasia renal es un hecho inusual (3). Este artículo presenta el caso de una paciente joven con un nódulo renal persistente en los exámenes de imagen tras 3 meses de tratamiento corticoterápico para Enfermedad de Kimura y fue encaminada a un tratamiento quirúrgico del nódulo del riñón.

\section{PRESENTACIÓN}

Se trata de una paciente del sexo femenino, 23 años de edad, con un cuadro súbito de linfadenomegalia cervical y submadibular, asociado a fiebre y dolor abdominal. La biopsia de linfonodos reveló linfadenítis granulomatosa supurativa con intensa eosinofília (Figura 1). La evaluación inmunohistoquímica mostró positividad para células de Reed-Sternberg. La tomografía computerizada de abdomen y tórax mostró linfonodos aumentados de tamaño, y masas en el mediastino, periesplénicas y perirrenales bilaterales. Se notó un nódulo más evidente de aproximadamente $35 \mathrm{~mm}$ en el polo inferior del riñón izquierdo (Figura 2). La función renal estaba preservada. Los exámenes de laboratorio mostraron eosinofilia y aumento de inmunoglobina $\mathrm{E}$.

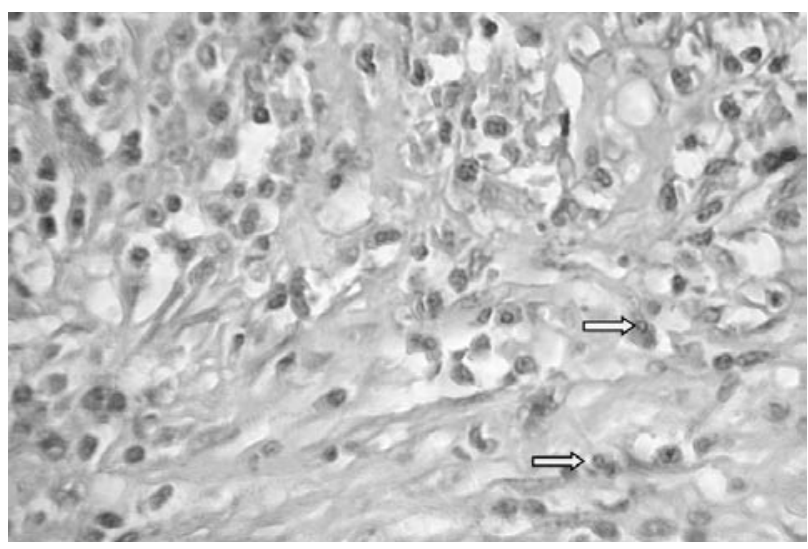

FIGURA 1. Histología del linfonodo submandibular, coloración con Hematoxilina y Eosina laumento de 400 veces). Predominio de eosinófilos (flechas).
El tratamiento con corticoide, $20 \mathrm{mg}$ de prednisona, durante 10 semanas mejoró el cuadro de las nodulaciones, linfadenomegalias, dolor abdominal y fiebre. Los niveles, de eosinofilia e inmunoglobulinas $E$, retrocedieron hacia los valores basales. Tras tres meses de corticoterapia la tomografía computadorizada de abdomen mostró persistencia del nódulo renal del lado izquierdo. La paciente fue sometida a una nefrectomía parcial izquierda. El examen histológico reveló carcinoma renal de células raras. Tras dieciocho meses, la paciente se encuentra sin signos o síntomas de la Enfermedad de Kimura o de rediciva de la enfermedad neoplásica renal.

\section{PACIENTE Y MÉTODO}

Se presenta el relato del caso de una paciente del sexo femenino con enfermedad de Kimura y neoplasia epitelial renal, además de una revisión de la literatura médica en los bancos de datos medline y bireme.

\section{DISCUSIÓN}

La Enfermedad de Kimura (Síndrome de Kimura o Linfogranuloma eosinofílico) es una condición clínica diagnosticada en la mayoría de las veces en una propedéutica para linfonodos cervicales aumentados (3). La causa de la enfermedad parece todavía ser desconocida (24). Histológicamente, la lesión se caracteriza por hiperplasia linfoide, con presencia sobresaliente de un gran número de eosinófilos (5), y proliferación de capilares. Esa enfermedad puede mimetizar un proceso neoplásico (2) y debe tener como diagnóstico diferencial linfomas, fibrohistiocitoma maligno, linfoepitelioma benigno (5), neoplasia de glándula parótida (2) y otros. El principal diagnóstico diferencial de la Enfermedad de Kimura, no obstante, es la hiperplasia angiolinfoide con eosinofilia

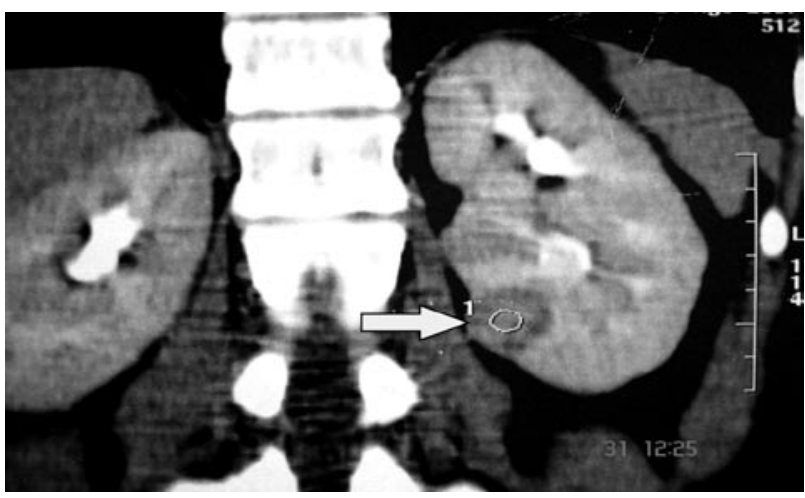

FIGURA 2. Tomografía computadorizada de abdomen. Nódulo en el riñón izquierdo (flecha). 
(5). En el presente caso la biopsia de los linfonodos cervicales submandibulares mostraron inicialmente linfadenitis reaccional no específica, con predominio de eosinófilos. La inmunohistoquímica contribuyó para el diagnóstico definitivo, pues fue positivo el examen para células de Reed-Stenberg, que es bastante sugerente de Enfermedad de Kimura (1).

El tratamiento de la Enfermedad de Kimura debe hacerse con corticoides, como por ejemplo, la prednisona. Se recomienda aguardar alrededor de seis semanas tras el inicio de la corticoterapia para abordar quirúrgicamente las masas o nódulos residuales (2). En el caso del abordaje operatorio del nódulo renal izquierdo, del caso en cuestión, esperamos alrededor de 8 semanas para la realización de nuevos exámenes de imagen y, a continuación, se propuso la nefrectomía parcial izquierda. Pese a que los trabajos más recientes demuestran ser la exactitud diagnóstica de la biopsia renal percutánea superior al $90 \%(6,7)$ la misma no se viene realizando rutinariamente, principalmente debido a discusiones en cuanto al muestreo del material, alto índice de falsos negativos e implante neoplásico en el local de acceso (8). En el único artículo de la literatura que nombra un caso de neoplasia de riñón asociada a la Enfermedad de Kimura la biopsia de riñón fue conclusiva (3).

La indicación de nefrectomía se basó principalmente en las características tomográficas de la lesión, que sugerían neoplasia de origen maligno. La opción por cirugía preservadora de nefrones, nefrectomía parcial, se basó en el tamaño y ubicación periférica del nódulo, lo cual condice con la literatura actual (9).

Existe una asociación entre la enfermedad de Kimura y el síndrome nefrótico (5), aunque en este caso la función renal fuera normal.

La radioterapia puede recomendarse para lesiones nodulares subcutáneas o masas refractarias a la corticoterapia (4). El pronóstico de la enfermedad de Kimura suele ser bueno, con pocas recidivas.

Éste es el segundo caso en la literatura médica que describe la asociación entre la Enfermedad de Kimura con la neoplasia renal. Este caso puede reforzar la posible asociación entre Enfermedad de Kimura y otras patologías, aún no muy bien conocidas. Se puede suponer que el nódulo renal que persistió tras la corticoterapia sea una degeneración de un nódulo inflamatorio del Síndrome de Kimura. Del mismo modo, tal vez pueda ser el Síndrome de Kimura una manifestación paraneoplásica de una neoplasia primaria inicialmente no identificada. Además, también se puede inferir que el síndrome de Kimura puede ser sólo un período de inmunosupresión relativa, donde hay una superproducción de inmunoglobulina $E$ y eosinófilos, en medio a un proceso inflamatorio sistémico. En este caso, la paciente evolucionó bien, sin signos de masas o nódulos subcutáneos, mediastinales o abdominales residuales. Los valores de eosinófilos e inmunoglobulina $E$ regresaron al valor normal. No hubo signos de recidiva de la enfermedad neoplásica renal, tras 18 meses de seguimiento.

\section{CONCLUSIÓN}

La enfermedad de Kimura es una dolencia que tiene como una de las manifestaciones iniciales el aumento del volumen de linfonodos cervicales. La enfermedad está asociada a algunas neoplasias, incluso la de riñón. La nefrectomía parcial es una buena opción para el tratamiento del nódulo en el polo inferior del riñón.

\section{BIBLIOGRAFÍA Y LECTURAS RECOMENDADAS ( ${ }^{*}$ lectura de interés $y^{* *}$ lectura fundamental)}

*1. Chen H, Thompson LDR, Aguilera NSI, Abbondanzo SL. Kimura Disease. A clinicopathologic study of 21 cases. Am J Pathol, 2004; 28: 505-13.

2. Jani A, Coulson M. Kimura's Disease: a typical case of a rare disorder. West J Med, 1997; 166: 142-3.

**3. Akhavan A, Cannon Jr GM, Sasatomi E, Franks ME. Synchronous unilateral renal cell carcinoma and kimura disease of the kidney. Urol, 2006; 68: 673e21 -2 .

4. Larroche C, Bletry O. Kimura's disease. Orphanet encyclopedia; 2005: 1-3.

**5. Li TJ, Chen X M, Wang S Z, Fan M W, Semba I, Kitano M. Kimura's Disease. A clinicopathologic study of 54 chinese patients. Surg Oral Pathol Oral Radiol endod 1996;82: 549-55.

6. Volpe A, Kachura JR, Geddie WR, Evans AJ, Gharajeh A, Saravanan A and Jewett MA. Techniques, safety, and accuracy of sampling of renal tumors by fine needle aspiration and core biopsy. J Urol, 2007; 178: 379.

7. Beland MD, Mayo-Smith WW, Dupuy DE, Cronan JJ, DeLellis RA. Diagnostic of 58 consecutive imaging-guided biopsies of solid renal masses: should we biopsy all that are indeterminate? Am J Roent, 2007; 188: 792-7.

8. Bosniak MA. The small ( $<3,0 \mathrm{~cm}$ ) renal parenchymal tumor: detection, diagnosis, and controversies. Radiol, 1991; 179: 307-17.

9. Heldwein FL, McCullough TC, Souto CAV, Galiano M, Barret E. Localized renal cell carcinoma management: an update. Int Braz J Urol, 2008; 34: 676-90. 